\title{
The Outcome of Intracytoplasmic Injection of Testicular Spermatozoa and Epididymal Spermatozoa Obtained from Azoospermic Men
}

\author{
Nguyen Dinh Tao ${ }^{1}$, Trinh The Son ${ }^{1}$, Quan Hoang Lam ${ }^{1}$, Nguyen Thanh Tung ${ }^{1}$, Vu Van Tam ${ }^{2}$ and Hoang Van Luong ${ }^{*}$ \\ ${ }^{1}$ Vietnam Military Medical University, Ha Noi, Vietnam \\ ${ }^{2}$ Hai Phong IVF Center, Vietnam
}

*Corresponding author: Hoang Van Luong, Vietnam Military Medical University, Ha Noi, Vietnam, E-mail: luonghv@vmmu.edu.vn

Received date: July 19, 2016, Accepted date: August 03, 2016, Published date: August 10, 2016

Copyright: (c) 2016 Tao DN, et al. This is an open-access article distributed under the terms of the Creative Commons Attribution License, which permits unrestricted use, distribution, and reproduction in any medium, provided the original author and source are credited.

\begin{abstract}
This study was carried out to compare the reproductive capability of testicular sperm injection (ICSI) technique. From November 2006 to December 2007, 198 intracytoplasmic sperm injection cycles were studied. One hundred thirty of 198 cycles were carried out using epididymal sperm and 68 were carried out using testicular sperm. The results showed that no significant differences were observed in the rates of fertilization and embryo transfer between obstructive and nonobstructive etiology and between epididymal and testicular spermatozoa. The abortion rate of motile epididymal or testicular spermatozoa was lower than that of immotile spermatozoa.
\end{abstract}

Keywords: Azoospermia; ICSI; Epididymis; Fertility; Male; Testis

\section{Introduction}

A common etiology of azoospermia in infertile men was surgically unreconstructable obstruction of the male genital tract [1]. Historically, a patient was considered infertile with obstructive azoospermia caused by congenital absence of the vas deferens (CAVD) or those had failed in reconstructive surgery. However, recent studies show that it is worthwhile to offer new surgical reconstruction to patients in whom previous seminal tract reconstructive surgery failed [2]. After the first successful pregnancies and the success reported using intracytoplasmic sperm injection (ICSI) in patients with severe oligoasthenoteratozoospermia the treatment of azoospermic infertile patients changed $[3,4]$. Actually, this novel therapy relating to retrieval of spermatozoa by surgery combined with aided reproduction has provided hopes to patients considered previously as an untreatable infertile. Spermatozoa retrieved by microsurgical aspiration of epididymal sperm (MESA) have been used for more than a decade for in vitro fertilization (IVF) [5]. Silber et al. first documented conception with sperm aspirated from the head of the epididymis and IVF in cases of bilateral CAVD [6]. The fertilization and pregnancy rates with MESA and standard IVF are low at not higher than $20 \%$ and $11 \%$, respectively [5,7]. Yet, the micromanipulation for aided fertilization has improved the rate of fertilization significantly [8,9]. Moreover, ICSI may be considered an effective fertility treatment for patients with nonobstructive azoospermia. In nonobstructive origin azoospermia patient, the sperm cells can be taken only from testicles by testicular sperm aspiration (TESA) [10-14]. The fertilization and pregnancy rates of ICSI have been assessed in several studies [15-24]. Because of the contradictions in published reports, which may be partially due to sperm unrelated factors, the impact of the source of spermatozoa (testicular versus epididymal) and the etiology of azoospermia (obstructive versus nonobstructive) on sperm reproductive capacity is not well established. The ICSI result in obstructive azoospermia group was compared with nonobstructive azoospermia group, epididymal azoospermia group was compared with testicular azoospermia, and motile was compared with immotile spermatozoa.

\section{Materials and Methods}

\section{Data collection}

Our retrospective study between November 2006 and December 2007 included 60 consecutive azoospermic patients in 62 ICSI cycles. In 198 cycles, 68 cases were manipulated in nonobstructive azoospermic patients and 130 cases were carried out in obstructive azoospermic patients. 52 cycles ( 40 patients) were performed using percutaneous epididymal sperm aspiration (PESA), 130 cycles (110 patients) were handled using TESA and 16 cycles (16 patients) were manipulated using PESA plus TESA. Sperm was taken from the testis until 1998 in obstructive azoospermia patients. After that, spermatozoa were retrieved from the epididymis in obstructive azoospermia patients and spermatozoa were retrieved from the testicles in nonobstructive azoospermia patients. Nonobstructive azoospermia was diagnosed by testis biopsy showing spermatogenesis failure. In obstructive azoospermia patients with surgically irreparable obstruction, CAVD or previous vasectomy reversal failure and in those who did not elect vasectomy reversal, ICSI was performed. Female partners were younger than 39 years and baseline serum folliclestimulating hormone (FSH) was lower than $10 \mathrm{mIU} / \mathrm{ml}$ in all cases. Complete history, physical examination and hormone evaluation were performed. Patients with nonobstructive azoospermia and abnormal karyotype examination of peripheral leukocytes were excluded from study. Patients were informed about the ICSI technique using epididymal and testicular spermatozoa, and possible complications of the surgical procedures, such as infection, hematoma and impaired blood flow. All patients were counseled and signed a consent form approved by our internal ethics committee.

\section{Ovarian stimulation and oocyte retrieval}

Infertility evaluation in female partner consisted of assessment and hysterosalpingography. Average age of the female partners was $32.3 \pm$ 5.5 years. No statistically significant differences in average female age in the obstructive and nonobstructive azoospermia groups ( $33.5 \pm 4.2$ and $34.2 \pm 3.8$ years, respectively) were observed. A unique protocol of 
Citation: Tao ND, Son TT, Lam QH, Tung NT, Tam VV, et al. (2016) The Outcome of Intracytoplasmic Injection of Testicular Spermatozoa and Epididymal Spermatozoa Obtained from Azoospermic Men. J Aging Sci 4: 157. doi:10.4172/2329-8847.1000157

Page 2 of 5

controlled ovarian stimulation including gonadotropin-releasing hormone agonist (leuprolide acetate) for at least 14 days was performed in female patients. When serum estradiol concentrations were less than $40 \mathrm{pg} / \mathrm{ml}$ and there are no ovarian cystic structures on ultrasound image, FSH was used for ovarian stimulation in a stepdown protocol to achieved at least 2 follicles with average diameter of $18 \mathrm{~mm}$. 36 hours after the administration of 10,000 IU human chorionic gonadotropin, oocyte was taken under transvaginal ultrasonography. Human tubular fluid medium added with $7.5 \%$ synthetic serum was used for oocytes preserving for approximately 3 to5 hours. After that, the cumulus cell was removed from the oocytes and placed in hyaluronidase at a concentration of $80 \mathrm{IU} / \mathrm{ml}$ for about 30 to 60 seconds. Then, oocytes were taken to fresh medium. Corona cells were removed by gently pipetting in and out.

\section{ICSI procedure}

ICSI was carried out with oocytes in metaphase II according to the method described by Palermo et al. ICSI was done with a heated microscope stage. After being injected, the oocytes were rinsed and placed in suitable growing medium. These oocytes were then incubated in a culture dish. About 16 to 18 hours after the injection procedure, the oocytes were checked for fertilization. Healthy oocytes were kept otherwise were removed from the dish. The fertilized oocytes were assessed after 24 hours cleavage. Embryo was transfered about 72 hours after injection.

\section{Sperm retrieval}

On the day of ovum retrieval percutaneous PESA or TESA was performed. All procedures were done in our outpatient procedure room using local anesthesia. Povidone-iodine was used for the area after shaving of the anterior scrotum. Lidocaine $1 \%$ was administered for anesthesia in the pubic tubercle area when no sperm was obtained. The testicle was immobilized for PESA by holding the inferior two thirds of the testicle and pulling the epididymis. The epididymis carefully stabilized between the thumb and index finger by surgeon to expose the superior pole of the patient's testicle. The suction of sperm was performed by a 23 gauge butterfly needle inserted into the epididymis and pulling back the plunger of the $20 \mathrm{cc}$ syringe. When the plunger reached $20 \mathrm{cc}$, the butterfly tubing was clamped at a point closed to the hub of the syringe. This made the $20 \mathrm{cc}$ syringe replacement easier with a $1 \mathrm{cc}$ tuberculin syringe without releasing negative pressure. Massaged the epididymis to knead fluid into the tubing. When the fluid return stopped, the needle was removed. Sperm was collected in similar fashion for TESA. A 19 gauge butterfly needle was used. The surgeon immobilized the testicle by grasping the epididymis and cord between the fingers, while pulling the scrotal skin taut. The depth of the needle excursion was controlled to protect the epididymis from injury. Inserted the needle into the inferior pole of the testis, and rapidly advanced and pulled back several times toward the superior pole. The seminiferous tubules were aspirated by withdrawing the needle slightly and redirected several times to disrupt the testicular architecture. Repeated the procedure until an opaque to yellow fluid and tissue was flowed into the butterfly tubing. Washed the aspirate within the needle or proximal tubing with a small volume of culture medium into a Falcon tube maintained at $37^{\circ} \mathrm{C}$. The testicular tissue was minced into small pieces with sterilized scissors and forced through a 25 gauge needle. The homogenized tissue was washed twice with culture medium and the pellets were suspended and recovered. Postoperative pressure was kept constantly on the aspiration site in the operating room for 5 minutes. After that, a fluff compression dressing and scrotal supporter were applied and left in place for 24 hours. Student's $t$, the chi-square and Fisher's exact tests was used for statistical significance evaluation. Statistically significant differences were considered at $\mathrm{p} \leq 0.05$.

\section{Results}

No significant differences in the mean age of patients with obstructive and nonobstructive azoospermia were observed (32.2 \pm 3.7 and $30.6 \pm 5.1$ years, respectively, $\mathrm{p} \geq 0.56$ ). Of the 198 cycles 68 $(34.3 \%)$ and $130(65.6 \%)$ were performed in nonobstructive and obstructive azoospermia cases, including $71 \%$ and $28.4 \%$ of obstructive azoospermia cases involving vasectomy and nonvasectomy conditions (epididymis obstruction, CAVD and herniorraphy), respectively. ICSI using motile spermatozoa was done in $71.7 \%$ of the cycles, while immotile spermatozoa were retrieved and injected in $17.2 \%$.

\begin{tabular}{|l|l|l|l|}
\hline & Obstructive Azoospermia (1) & Non-obstructive Azoospermia (2) & P1-2 \\
\hline Mean age \pm SD & $32.2 \pm 3.7$ & $30.6 \pm 5.1$ & 0.56 \\
\hline \% Normal fertilization (2 PN) & 60.5 & 54 & 0.4 \\
\hline \% Abnormal fertilization (1,3 PN) & 16.6 & 16.4 & 0.5 \\
\hline \% Embryo transfer & 73.6 & 74.2 & 22 \\
\hline \% Pregnancy/cycle & 30 & 23.8 & 0.5 \\
\hline \% Pregnancy/transfer & 32.5 & 28.3 & 0.04 \\
\hline \% Pregnancy/patient & 39.8 & 40 & 0.03 \\
\hline \% Abortion & 28 & & 0.03 \\
\hline PN: Pronucleus & & 0.01 \\
\hline
\end{tabular}

Table 1: Fertility outcome after ICSI in azoospermic patients 
Citation: Tao ND, Son TT, Lam QH, Tung NT, Tam VV, et al. (2016) The Outcome of Intracytoplasmic Injection of Testicular Spermatozoa and Epididymal Spermatozoa Obtained from Azoospermic Men. J Aging Sci 4: 157. doi:10.4172/2329-8847.1000157

Page 3 of 5

Spermatogenic cells, namely round and elongated spermatids, were used in $9.1 \%$ and $2.01 \%$ of cases, respectively. The abnormal fertilization ( 1 plus 3 pronuclei), normal fertilization ( 2 pronuclei), and embryo transfer rates for obstructive and nonobstructive azoospermia were $16.6 \%, 60.5 \%$, and $73.6 \%$, and $16.4 \%, 54 \%$, and $74.2 \%$, respectively $(\mathrm{p}>0.05$; Table 1$)$.

For obstructive azoospermia, the pregnancy rate per cycle, per transfer and per patient, and the abortion rate were $30 \%, 32.5 \%, 39.8 \%$ and $2 \%$, respectively, and for nonobstructive azoospermia, these rates were $22 \%, 23.8 \%, 28.3 \%$ and $40 \%$, respectively ( $\mathrm{p}<0.05$ ). For PESA, the normal, abnormal fertilization, and embryo transfer rates were $58.7 \%$, $21.4 \%$ and $73.6 \%$, respectively. For PESA plus TESA, these rates were $62.3 \%, 10.4 \%$ and $78 \%$, respectively. For TESA, these rates were, $57.3 \%$, $14.5 \%$ and $73.4 \%$, respectively ( $\mathrm{p}>0.05$, Table 2 ).

No significant differences in mean patient age in the 3 groups were observed $(31.4,32.7$ and 30.8 years, $\mathrm{p}>0.05)$. For PESA, the pregnancy rate per cycle, per transfer and per patient, and the abortion rate were $34.6 \%, 40 \%, 54.5 \%$ and $11.1 \%$, respectively. For PESA plus TESA these rates were, $37.5 \%, 37.5 \%, 37.5 \%$ and $33.3 \%$, respectively. For TESA these rates were $26.1 \%, 28.3 \%, 31 \%$ and $41 \%$, respectively (PESA versus PESA plus TESA $\mathrm{p}>0.05$, and PESA and PESA plus TESA versus TESA $p>0.05$ ). The pregnancy and miscarriage rates as well as the type of injected spermatozoa were shown on Table 3. No significant differences were demonstrated in the pregnancy rate per cycle and per patient for motile (41.04\% and $45.7 \%$ ) and immotile $(29.4 \%$ and 36.8 $\%$, respectively) sperm ( $>0.05)$. The lowest pregnancy rate per cycle and per patient was in patients in whom round and elongated spermatids were retrieved ( $15 \%$ and $13.63 \%$, respectively). The abortion rate was higher in those in whom immotile versus motile spermatozoa were retrieved $(70 \%$ versus $25.5 \%, \mathrm{p}<0.05)$. The round and elongated spermatids gave $100 \%$ rate of miscarriage.

\begin{tabular}{|l|l|l|l|l|}
\hline Variables & PESA(1) & PESA-TESA(2) & TESA(3) & p \\
\hline Mean age & 31.4 & 32.7 & 30.8 & P1-2; P2-3; P 3-1 $\geq 0.05$ \\
\hline \%Normal fertilization (2 PN) & 58.7 & 62.3 & 57.3 & P1-2; P2-3; P 3-1 $\geq 0.05$ \\
\hline \%Abnormal fertilization (1,3 PN) & 21.4 & 10.4 & 14.5 & P1-2; P2-3; P 3-1 $\geq 0.05$ \\
\hline \%Embryo transfer & 73.6 & 78 & 73.4 & P1-2; P2-3; P 3-1 $\geq 0.05$ \\
\hline \%Pregnancy/cycle & 34.6 & $37.5^{*}$ & 26.1 & P1-2; P2-3 $\leq 0.05$ \\
\hline \%Pregnancy/transfer & 40 & $37.5^{*}$ & 28.3 & P1-2; P2-3 $\leq 0.05$ \\
\hline \% Pregnancy/patient & 54.5 & $37.5^{*}$ & 31 & P1-2; P2-3 $\leq 0.05$ \\
\hline \% Abortion & 11.1 & $33^{*}$ & 41 & P1-2; P2-3 $\leq 0.05$ \\
\hline
\end{tabular}

Table 2: Fertility outcome in patients with epididymal and testicular spermatozoa

\begin{tabular}{|l|l|l|l|}
\hline & Round - Elongated Spermatids & Motile Spermatozoa & Immotile Spermatozoa \\
\hline No. obstructive azoospermia (\%) & $3(2.3)$ & $107(82.3)$ & $20(15.4)$ \\
\hline No. nonobstructive azoospermia (\%) & $19(27.9)$ & $35(51.47)$ & $14(20.58)$ \\
\hline No. cycles & 22 & 142 & 34 \\
\hline No. pregnancies/cycle (\%) & $3(13.63)$ & $55(41.04)$ & $10(29.4)$ \\
\hline No. pts. & 20 & 127 & 19 \\
\hline No. pregnancies/pt. & $3(15)$ & $58(45.7)$ & $7(36.8)$ \\
\hline No. abortions (\%) & $3(100)$ & $14(25.5)$ & $7(70)$ \\
\hline
\end{tabular}

Table 3: Outcome according to azoospermia etiology and the results of spermatozoa retrieval

\section{Discussion}

New technique of testicular sperm aspiration combined with ICSI have been developed since the introduction of MESA for obstructive azoospermia caused by bilateral CAVD [6]. Consequently, many azoospermic patients with various causes can be offered IVF. As a result of the high fertilization and pregnancy rates that may be achieved by ICSI, this technology is now routinely used to improve the results of IVF after epididymal sperm aspiration. For couples with infertility secondary to nonobstructive azoospermia no corrective treatment is available except hypogonadotropic hypogonadism. However, some of these couples were able to have genetic offspring using the small number of spermatozoa in the testicles with ICSI. It seems that these azoospermic patients have small foci of spermatogenesis in the testes. It was estimated that there must be at least 4 to 6 mature spermatids per tubule for any spermatozoa to reach 
the ejaculate $[25,26]$. Conventionally, azoospermic patients with serum FSH greater than 2 to 3 -fold normal, who have severe testicular failure were not amenable to any conventional therapy. However, testicular sperm retrieval resulted in a lower pregnancy rate as well as a higher abortion rate than epididymal sperm retrieval. Our study demonstrated that the normal fertilization rate (2 pronuclei) in nonobstructive azoospermic patients was $54 \%$, similar to the normal fertilization rate achieved in obstructive azoospermic patients (60.5\%). Also, fertilization rates were similar for epididymal and testicular spermatozoa. The reason why there were no differences in the fertilization rate in patients with obstructive (epididymal spermatozoa) and nonobstructive azoospermia (testicular spermatozoa) was not known. However, the pregnancy rate per cycle and per patient in patients with nonobstructive was significantly lower than those with obstructive azoospermia. We also noted that the pregnancy rate was higher for epididymal versus testicular spermatozoa. Furthermore, testicular spermatozoa were related to a higher abortion rate than epididymal spermatozoa. Actually, there is severe impairment of spermatogenesis and even testicular failure nonobstructive azoospermic patients [27]. Nonobstructive azoospermic patients may have a defect in genes or a genetically determined barrier inhibited reproduction. Therefore, it is reasonable that despite successfully extracting live spermatozoa in, the pregnancy rate of nonobstructive azoospermic patients was significantly lower than that of obstructive azoospermic patients. The fertilization rate of ICSI was similar in patients with obstructive (epididymal) and nonobstructive (testicular) spermatozoa in this retrospective analysis. In our study, testicular spermatozoa resulted in a higher abortion rate than epididymal spermatozoa. In contrast to the findings of Silber et al. [28], although similar fertilization and cleavage can be obtained in epididymal and testicular spermatozoa, there was still different in pregnancy rates. However, the pregnancy rates can be determined by other factors other than spermatozoa, such as maternal age and ovarian reserve thus impairing the outcome [28]. As described, the higher miscarriage rate associated with testicular sperm may be associated with azoospermia etiology. These cells probably carry genetic disorders preventing pregnancy establishment, mainly in nonobstructive azoospermia cases. Genetically defective sperm can lead to fetal loss and genetic disease in the offspring $[29,30]$. We also found out that motile spermatozoa had lower abortion rate than immotile spermatozoa. Sperm acquire the capacity for vigorous forward motility during transit through the epididymis, which has an important role in sperm maturation [31]. During this process, the function of the sperm centrosome may be affected by some alterations resulting in impaired motility and a low percent of vital sperm.

\section{Conclusions}

Our study shows that the fertilization rate of ICSI is similar in patients with obstructive (epididymal) and nonobstructive (testicular) spermatozoa. Testicular spermatozoa are associated with a higher abortion rate than epididymal spermatozoa. Also, the abortion rate of motile epididymal or testicular spermatozoa was lower than that of immotile spermatozoa.

\section{References}

1. Patricio P, Ord T, Silber SJ (1988) Two births after microsurgical epididymal sperm aspiration in congenital absence of the vas deferens. Lancet 2: 1364.

2. Pasqualotto FF, Agarwal A, Srivastava M (1999) Fertility outcome after repeat-vasoepididymostomy. J Urol 162: 1626-1628.
3. Palermo G, Joris H, Devroey P (1992) Pregnancy after intracytoplasmic injection of a single spermatozoon into an oocyte. Lancet 304: 17-18.

4. Van Steirteghem AC, Nagy Z, Joris H (1993) High fertilization and implantation rates after intracytoplasmic sperm injections. Hum Reprod 8: 1061-1066.

5. Temple-Smith PD, Southwick GJ, Yates CA (1985) Human pregnancy by in vitro fertilization (IVF) using sperm aspirated from the epididymis. J In Vitro Fertil Embryo Transfer 2: 119-122.

6. Silber SJ, Nagy ZP, Liu J (1994) Conventional in-vitro fertilization versus intracytoplasmic sperm injection for patients requiring microsurgical sperm aspiration. Hum Reprod 9: 1705-1709.

7. Silber SJ, Asch R, Balmaceda J (1988) Pregnancy with sperm aspiration from the proximal head of the epididymis: a new treatment from congenital absence of the vas deferens. Fertil Steril 50: 525-528.

8. choysman R, Vanderwalmen P, Nijs (1993) Pregnancy after fertilization with human testicular spermatozoa. Lancet 342: 1237.

9. Assisted reproductive technology in the United States (2000) results generated from the American Society for Reproductive Medicine/Society for Assisted Reproductive Technology Registry. Society for Assisted Reproductive Technology and American Society for Reproductive Medicine. Fertil Steril 74: 641.

10. Belker AM, Sherins RJ, Dennison-Lagos L (1998) Percutaneous testicular sperm aspiration: a convenient and effective office procedure to retrieve sperm for in vitro fertilization with intracytoplasmic sperm injection. J Urol 160: 2058-2062.

11. Schlegel PN (1999) Testicular sperm extraction: microdissection improves sperm yield with minimal tissue excision. Hum Reprod 14: 131-135.

12. Turek PJ, Cha I, Ljung BM (1997) Systematic fine-needle aspiration of the testis: correlation to biopsy and results of organ "mapping" for mature sperm in azoospermic men. Urology 49: 743.

13. Silber SJ (2000) Microsurgical TESE and the distribution of spermatogenesis in non-obstructive azoospermia. Hum Reprod 15: 2178-2284.

14. Ezeh UI, Moore HD, Cooke ID (1998) A prospective study of multiple needle biopsies versus a single open biopsy for testicular sperm extraction in men with non-obstructive azoospermia. Hum Reprod 13: 3075-3080.

15. Meng MV, Cha I, Jung BM (1973) Relationship between classic histological pattern and sperm findings on fine needle aspiration map in infertile men. Hum Reprod 15: 1973-1977.

16. Tournaye H (1999) Surgical sperm recovery for intracytoplasmic sperm injection: which method is to be preferred?. Hum Reprod 14: 71-81.

17. Craft I, Tsirigotis M, Bennet V (1995) Percutaneous epididymal sperm aspiration and intracytoplasmic sperm injection in the management of infertility due to obstructive azoospermia. Fertil Steril 63: 1038-1042.

18. Mansour RT, Kamal A, Fahmy I (1997) Intracytoplasmic sperm injection in obstructive and nonobstructive azoospermia. Hum Reprod 12: 1974.

19. Gil-Salo M, Romero J, Mýnnguez Y (1998) Testicular sperm extraction and intracytoplasmic sperm injection: a chance of fertility in nonobstructive azoospermia. J Urol 160: 2063-2067.

20. Palermo GD, Schlegel PN, Hariprashad JJ (1999) Fertilization and pregnancy outcome with intracytoplasmic sperm injection for azoospermic men. Hum Reprod 14: 741-748.

21. De Croo I, Van der Elst J, Everaert K (2000) Fertility, pregnancy and embryo implantation rates after ICSI in cases of obstructive and nonobstructive azoospermia. Hum Reprod 15: 1383-1388.

22. Ubaldi F, Nagy ZP, Rienzi L (1999) Reproductive capacity of spermatozoa from men with testicular failure. Hum Reprod 14: 2796-2800.

23. Madgar I, Horvitz A, Levron J (1998) Outcome of in vitro fertilization and intracytoplasmic injection of epididymal and testicular sperm extracted from patients with obstructive and nonobstructive azoospermia. Fertil Steril 69: 1080-1084.

24. Mercan R, Urman B, Alatas C (2000) Outcome of testicular sperm retrieval procedures in non-obstructive azoospermia: percutaneous aspiration versus open biopsy. Hum Reprod 15: 1548-1551. 
Citation: Tao ND, Son TT, Lam QH, Tung NT, Tam VV, et al. (2016) The Outcome of Intracytoplasmic Injection of Testicular Spermatozoa and Epididymal Spermatozoa Obtained from Azoospermic Men. J Aging Sci 4: 157. doi:10.4172/2329-8847.1000157

Page 5 of 5

25. Silber SJ, Nagy Z, Devroey P (1997) Distribution of spermatogenesis in the testicles of azoospermic men: the presence or absence of spermatids in the testes of men with germinal failure. Hum Reprod 12: 2422-2428.

26. Kim ED, Gilbaugh JH, Patel VR (1997) Testis biopsies frequently demonstrate sperm in men with azoospermia and significantly elevated follicle-stimulating hormone levels. J Urol 157: 144-146.

27. Martin RH, Greene C, Rademaker A (2000) Chromosome analysis of spermatozoa extracted from testes of men with non-obstructive azoospermia. Hum Reprod 15: 1121-1124.

28. Silber SJ, Nagy Z, Devroey P (1997) The effect of female age and ovarian reserve on pregnancy rate in male infertility: treatment of azoospermia with sperm retrieval and intracytoplasmic sperm injection. Hum Reprod 12: $2693-2700$

29. Silber SJ, Nagy Z, Liu J (1995) The use of epididymal and testicular sperm for ICSI: the genetic implications for male infertility. Hum Reprod 10 2031-2043.

30. Silber SJ, Nagy Z, Liu J (1995) High fertilization and pregnancy rates after ICSI with spermatozoa obtained from testicle biopsy. Hum Reprod 10: $148-152$.

31. Nagy ZP (2000) Sperm centriole disfunction and sperm immotility. Mol Cell Endocr166: 59. 